\title{
IAPEX OF APVERTISERS
}

4pi Analysis, Inc (www.4pi.com)

Advanced Microscopy Techniques (www.amtimaging.com)

Ascend Instruments (www.ascendinstruments.com)

Bruker AXS Microanalysis (www bruker-axs.com

Bruker Optics, Inc. (www.brukeroptics.com/microscopy) ..

Buehler, LTD. (www.buehler.com)

Carl Zeiss SMT, Inc. (smt.zeiss.com)

Duniway Stockroom Corp. (www.duniway.com) .

E.A. Fischione, Inc (www.fischione.com)

e2v Technologies (enquiries@e2vtechnologies.com)

EBSciences (ebs@ebsciences.com).

EDAX Inc.(www.edax.com/integration)

Ernest F. Fullam (www.fullam.com)

Evex Analytical (www.evex.com)

FEI Company (www.feic.com)

Gatan Inc. (www.gatan.com)

Geller MicroAnalytical Lab (www.gellermicro.com)

Hitachi High Technologies America (www.hitachi-hhta.com) ........ 2, 5, 7, 9, 11

Hysitron, Inc. (www.hysitron.com)

Imago Scientific Instruments Corporation, (www.imago.com)

IXRF Systems (www.ixrfsystems.com)

JENOPTIK JENA (www.jenoptik-los.com)

JEOL USA (www.jeol.com)

LabX (www.labx.com)
55 M.E. Taylor Engineering (www.semsupplies.com)

45

75 Materials Analytical Services (MAS) (www.mastest.com) ...................... 21

79, 82 MAX Detector Repair Group LLC (www.maxdetector.com) .................. 89

47 Micro Optics of Florida, Inc. (info@microopticsfl.com) ......................... 59

.29 Minitool Micro Precision Products (www.minitoolinc.com) ................ 83

61 minus k TECHNOLOGY (www.minusk.com) ………………………..... 85

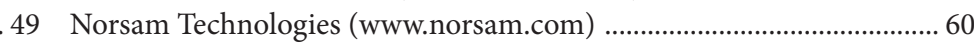

81 Omniprobe, Inc. (www.omniprobe.com) ……....................................... 51

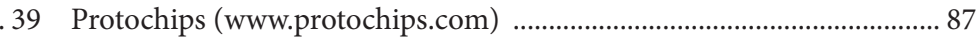

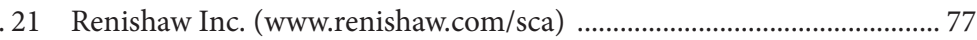

15, 59 Scientific Instruments and Applications (www.sia-cam.com) ................ 81

25 ScopeTronix Quality Products (www.scopetronix.com) ......................... 85

45 SEMTech Solutions (www.semtechsolutions.com) ................................... 37

53 SII NanoTechnology USA Inc. (www.siintusa.com) .............................. 89

41 Smart Imaging Technologies (info@smartimtech.com) .......................... 89

13 Soft Imaging Systems Corporation (www.soft-imaging.com) ............... 57

45 South Bay Technology, Inc. (www.southbaytech.com) …....................... 27

Ted Pella, Inc. (www.tedpella.com) ........................................................ 73

69 Tescan USA (www.tescan-usa.com) ......................................................... 71

.35 Thermo Electron Corp. - Molecular Spectroscopy (www.thermo.com) .... 43, 91

63 XEI Scientific (www.evactron.com) ..................................................... 65

85 Zyvex Corp. (www.zyvex.com) …………......................................... 31, 33

17, 19, 92 Employment Ads

23 Cabot Labs

\section{SUBSORIPTIOA REQUESTS/OHMAGES}

\section{Individuals requesting subscriptions, or wishing to change/correct their current address, are requested to do so via our $w w w$ site: \\ http://www.microscopy-today.com \\ SUBSCRIPTION POLICIES}

Microscopists in Canada, Mexico, and the United States of America are eligible for free subscriptions.

Microscopy Society of America members receive Microscopy Today as a free member benefit anywhere in the world.

- Non-MSA members, outside North America, may subscribe for \$50US per year to cover postage and handling.

- Scanned back issues of MT are available for low-resolution free download on our www site above, after a six-month delay. The entire issues are downloaded-advertisements and all.

- High resolution, $\mathrm{CD}$ format, complete volumes are available via airmail for the prices listed below. Note that a volume contains 6 issue for 2002 onwards and 10 issues for 1992 to 2001. Contact editor to order.

Preferred payment is via credit card information when subscribing at the www address. Bank transfer information available from the editor. Checks in US\$, payable to "Microscopy Today," to the editor.

\begin{tabular}{|l|c|c|c|}
\hline \multirow{2}{*}{$\begin{array}{c}\text { IIIOROSOPY TOPMY } \\
\text { Subscription Schedule }\end{array}$} & $\begin{array}{c}|c| \\
\text { Current Printed } \\
\text { Issue }\end{array}$ & $\begin{array}{c}\text { Back Issue } \\
\text { Downloaded After } \\
6-\text { Month Delay }\end{array}$ & $\begin{array}{c}\text { Back Issue Volume } \\
\text { High Resolution CD }\end{array}$ \\
\cline { 2 - 4 } Anyone in North America & Free & Free Low Resolution & $\begin{array}{c}\text { MSA member } \$ 25 / \text { volume } \\
\text { Non-MSA mbr. } \$ 40 / \text { volume }\end{array}$ \\
\hline MSA member outside North America & Free & Free Low Resolution & $\$ 35 /$ volume \\
\hline Non-MSA member, outside North America & $\$ 50 /$ year (6 issues) & Free Low Resolution & \$50/volume \\
\hline
\end{tabular}

